\title{
Abcb4 acts as multixenobiotic transporter and active barrier against chemical uptake in zebrafish (Danio rerio) embryos
}

Stephan Fischer ${ }^{1,2}$, Nils Klüver ${ }^{3}$, Kathleen Burkhardt-Medicke ${ }^{3,4}$, Mirko Pietsch ${ }^{3}$, Anne-Marie Schmidt ${ }^{3}$, Peggy Wellner ${ }^{3}$, Kristin Schirmer ${ }^{1,2,5}$ and Till Luckenbach ${ }^{3^{*}}$

\begin{abstract}
Background: In mammals, ABCB1 constitutes a cellular "first line of defense" against a wide array of chemicals and drugs conferring cellular multidrug or multixenobiotic resistance (MDR/MXR). We tested the hypothesis that an $A B C B 1$ ortholog serves as protection for the sensitive developmental processes in zebrafish embryos against adverse compounds dissolved in the water.

Results: Indication for ABCB1-type efflux counteracting the accumulation of chemicals in zebrafish embryos comes from experiments with fluorescent and toxic transporter substrates and inhibitors. With inhibitors present, levels of fluorescent dyes in embryo tissue and sensitivity of embryos to toxic substrates were generally elevated. We verified two predicted sequences from zebrafish, previously annotated as abcb1, by cloning; our synteny analyses, however, identified them as abcb4 and $a b c b 5$, respectively. The abcb1 gene is absent in the zebrafish genome and we explored whether instead Abcb4 and/or Abcb5 show toxicant defense properties. Quantitative real-time polymerase chain reaction (qPCR) analyses showed the presence of transcripts of both genes throughout the first 48 hours of zebrafish development. Similar to transporter inhibitors, morpholino knock-down of Abcb4 increased accumulation of fluorescent substrates in embryo tissue and sensitivity of embryos toward toxic compounds. In contrast, morpholino knock-down of Abcb5 did not exert this effect. ATPase assays with recombinant protein obtained with the baculovirus expression system confirmed that dye and toxic compounds act as substrates of zebrafish Abcb4 and inhibitors block its function. The compounds tested comprised model substrates of human $A B C B 1$, namely the fluorescent dyes rhodamine $B$ and calcein-am and the toxic compounds vinblastine, vincristine and doxorubicin; cyclosporin A, PSC833, MK571 and verapamil were applied as inhibitors. Additionally, tests were performed with ecotoxicologically relevant compounds: phenanthrene (a polycyclic aromatic hydrocarbon) and galaxolide and tonalide (two polycyclic musks).

(Continued on next page)
\end{abstract}

\footnotetext{
* Correspondence: till.luckenbach@ufz.de

${ }^{3}$ Department of Bioanalytical Ecotoxicology, UFZ - Helmholtz Centre for Environmental Research, 04318 Leipzig, Germany

Full list of author information is available at the end of the article
} 
(Continued from previous page)

Conclusions: We show that zebrafish Abcb4 is a cellular toxicant transporter and provides protection of embryos against toxic chemicals dissolved in the water. Zebrafish Abcb4 thus is functionally similar to mammalian ABCB1, but differs from mammalian ABCB4, which is not involved in cellular resistance to chemicals but specifically transports phospholipids in the liver. Our data have important implications: Abcb4 could affect bioavailability - and thus toxicologic and pharmacologic potency - of chemicals to zebrafish embryos and inhibition of Abcb4 therefore causes chemosensitization, that is, enhanced sensitivity of embryos to toxicants. These aspects should be considered in (eco)toxicologic and pharmacologic chemical screens with the zebrafish embryo, a major vertebrate model.

Keywords: Abcb4, Abcb5, Chemosensitization, Efflux transporters, Environment-tissue barrier, Multixenobiotic resistance, MXR, P-glycoprotein

\section{Background}

ATP-binding cassette $(\mathrm{ABC})$ transporters are one of the largest membrane protein super-families and are present in all biota from prokaryotes to mammals. They play a fundamental role in the transport of exogenous and endogenous compounds across inter- and intracellular boundaries. Malfunction of certain members of the ABC family causes various diseases, such as Tangier disease, cystic fibrosis and Dubin-Johnson syndrome [1]. Among transporters that lower the cellular load of various toxicants and drugs and thus maintain physiological integrity is $\mathrm{ABCB} 1^{\mathrm{a}}$ or $\mathrm{P}$-glycoprotein, the best studied $\mathrm{ABC}$ transporter thus far. It confers so-called multidrug resistance (MDR) by diverting a range of drugs away from their site of pharmacologic action, a central concern in drug design for cancer therapy. On the other hand, ABCB1-like activity that was found in a wide range of organisms provides protection from toxicants, granting so-called multixenobiotic resistance (MXR) [2]. Indeed, knock-out of $A b c b 1$ in mice led to an increased permeability of the blood-brain barrier for neurotoxic compounds, allowing for enhanced brain penetration and lethal effects [3]. Moreover, occurrence of aquatic species in polluted environments is linked to high expression and increased efflux activity of Abcb1 orthologs [4], which are constituents of the transporter-mediated "environment-tissue barrier" [5]. In fact, mammalian $\mathrm{ABCB} 1$ is regarded as the only $\mathrm{ABC}$ transporter that has no physiological function other than protection of cells against a wide range of chemicals [6]. In contrast, two other $A B C$ transporters that are structurally highly similar to $\mathrm{ABCB} 1, \mathrm{ABCB} 4$ and $\mathrm{ABCB} 5$, appear not to be involved in cellular protection against toxicants. ABCB4 has a specific physiological function in the liver and transports only particular compounds [7,8]. Overexpression of ABCB5 in certain cancer cells is associated with multidrug resistance [9], but it may not provide toxicant protection to cells in its normal function.

Ex utero embryo development is common among aquatic organisms and requires cellular adaptations affording explicit robustness and protection against adverse environmental impacts. These "orphan" embryos are literally flooded with a multitude of natural and man-made chemicals and efflux transporter proteins appear to form a primary defense mechanism that keeps these compounds out [10]. For instance, embryos of the echiuroid worm, Urechis caupo, which develop in mudflats containing metabolic products of bacteria and plants, showed high levels of ABCB1-like efflux activity [11].

The zebrafish (Danio rerio) embryo has emerged as a valuable vertebrate model in diverse fields, such as genetics and human disease, pharmacology and (eco)toxicology [12]. While it represents the most diverse group of vertebrates - fishes - it also offers the exquisiteness of fast development, access to genetic manipulation, comparability of organogenesis to higher vertebrates and transparency, along with suitability for high-throughput screening. For example, 309 chemicals were evaluated for potential human health and ecological effects as part of the U.S. Environmental Protection Agency's (EPA's) ToxCast $^{\text {TM }}$ phase-I program ([13]; [14]) and thousands of chemicals were screened to identify novel neuroactive drugs in a high-throughput behavioral assay with zebrafish embryos [15]. Moreover, developments are underway to establish the zebrafish embryo for rapid evaluation of the toxicity and bioaccumulation potential of the thousands of chemicals undergoing environmental risk assessment instead of relying on juvenile and adult fish [16]. The role of ABC transporters in the zebrafish developmental processes and in dealing with chemical exposure is, however, as yet unknown, and uptake and distribution of chemicals are generally assumed to be driven by passive diffusion.

We here demonstrate that the uptake of chemicals by zebrafish embryos can in fact be substantially influenced by $\mathrm{ABC}$ transporter activity. Throughout the first 48 hours of development, zebrafish embryos exhibit activity of an ABCB1-like efflux. Surprisingly, however, an $a b c b 1$ ortholog appears to be absent in zebrafish. We found gene expression of $a b c b 4$ and $a b c b 5$ in zebrafish 
embryos that both are structurally similar to mammalian $A b c b 1$ and we show that Abcb4, but not Abcb5, protein possesses functional properties of mammalian ABCB1, constituting an active barrier against chemical uptake and conferring resistance of embryos to $\mathrm{ABCB} 1$ substrates.

\section{Results and discussion}

\section{P-glycoproteins in zebrafish are $a b c b 4$ and $a b c b 5$} orthologs

Three structurally similar proteins, $\mathrm{ABCB} 1, \mathrm{ABCB} 4$ and $\mathrm{ABCB} 5$, are considered as "P-glycoproteins". The zebrafish possesses only two P-glycoprotein genes that, being annotated as $a b c b 1$ orthologs, were previously designated $a b c b 1 a$ and $a b c b 1 b ; a b c b 4$ and $a b c b 5$ were considered absent [17]. We confirmed the predicted cDNA sequences of both genes by cloning. Nucleotide sequence identity of the cDNAs is $59 \%$ and the deduced amino acid sequences show 50 to $64 \%$ identities with human ABCB1, ABCB4 and ABCB5 (Additional file 1: Table S1).
According to current annotations on the ensembl database [18] former $a b c b 1 a$ is designated $a b c b 5$ (ENSDARG00000021787) and former $a b c b 1 b$ is $a b c b 4$ (ENSDARG00000010936); an $A b c b 1$ ortholog is absent. Our synteny analyses of the respective chromosomal localizations of the genes in zebrafish are in line with the ensembl annotations and support that the zebrafish Pglycoproteins are not $a b c b 1$ orthologs. Human $A B C B 1$ and $A B C B 4$ are consecutively located, whereas in zebrafish there is only one P-glycoprotein in a corresponding chromosome region. This gene is in close proximity to $\mathrm{crot}$, resembling human $A B C B 4$ and $C R O T$ (Figure 1A). Furthermore, genes characteristic for the close vicinity of human and other vertebrate $A b c b 1$ genes, comprising rundc3b, slc25a40 and adam22, are located approximately 14 megabases $(\mathrm{Mb})$ away from this locus in zebrafish. In concordance with zebrafish, other fish species (Latimeria chalumnae, Gasterosteus aculeatus, Oryzias latipes, Gadus morhua) also possess only $a b c b 4$, but not $a b c b 1$ (Additional file 1: Figure S1),

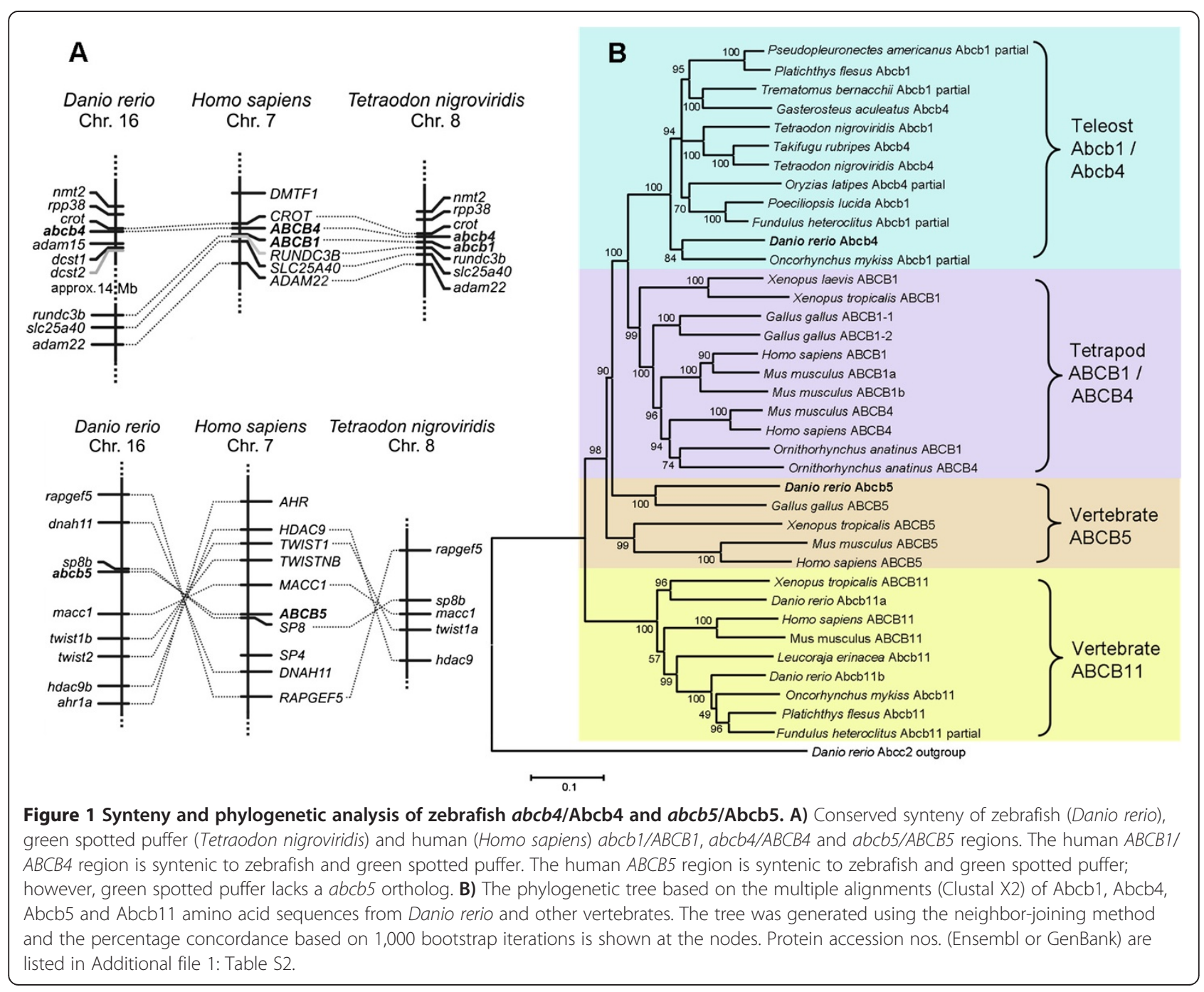


whereas Takifugu rubripes and Tetraodon nigroviridis have both $a b c b 1$ and $a b c b 4$ (Figure 1A, Additional file 1: Figure S1). Analysis of the $a b c b 1 a / a b c b 5$ chromosomal localization indicates that zebrafish $a b c b 1 a$ is an $a b c b 5$ ortholog. This chromosomal region of $a b c b 5$ shares synteny with human $A B C B 5$ (Figure 1A), indicating that this locus has a common ancestral origin. Zebrafish Abcb1a/Abcb5 protein also associates with Abcb5 orthologs from other organisms in a phylogenetic tree (Figure 1B).

In this tree, proteins from different fish species designated as "Abcb4", "Abcb1", "Mdr1" or "P-glycoprotein" group to a cluster of fish P-glycoproteins that show close relationship with tetrapod $\mathrm{ABCB} 1$ and $\mathrm{ABCB} 4$ (Figure 1B). This suggests that tetrapod and teleost $\mathrm{ABCB} 1 / \mathrm{Abcb} 1$ and ABCB4/Abcb4 have a common ancestor. Mammalian $\mathrm{ABCB} 1$ and $\mathrm{ABCB} 4$ sequences are highly similar but the functions of the proteins largely differ. Whereas substrate recognition of $\mathrm{ABCB} 1$ is rather unspecific, enabling it to act as a versatile efflux pump of toxicants, ABCB4 is a highly specialized transporter that trans-locates certain phospholipids and does not efflux toxicants from cells [7]. Differences in function despite the high sequence similarity of $\mathrm{ABCB} 1$ and $\mathrm{ABCB} 4$ raise the question if functional properties of the closely related fish P-glycoproteins are $\mathrm{ABCB} 1$ - or $\mathrm{ABCB} 4$-like and if they are toxicant transporters. Further, the ability of human ABCB5 to confer multidrug resistance of cancer cells [9] may suggest that the zebrafish Abcb5 ortholog may act as a cellular efflux pump of toxicants.

The absence of an $a b c b 1$ ortholog contradicted detection of an ABCB1-like efflux activity and multixenobiotic resistance of zebrafish embryos (see below). We, hence, investigated whether instead the other P-glycoproteins, Abcb4 and Abcb5, act as toxicant transporters.

\section{Abcb4 and $a b c b 5$ transcripts are constitutively expressed during the first $\mathbf{4 8}$ hours of zebrafish embryo development}

We examined constitutive expression levels of $a b c b 4$ and $a b c b 5$ transcripts in zebrafish embryos from 1 to 48 hpf (hours post fertilization) for determining if these genes are transcribed and thus are candidates to be involved in cellular toxicant defense in these stages. Transcripts of both transporters were present at all sampled time points of development (1, 6, 12, 24, $48 \mathrm{hpf})$ (Table 1). Abcb4 and $a b c b 5$ transcript levels varied one to two orders of magnitude among developmental stages, but expression was generally low in comparison to, for example, $\beta$-actin with transcript levels that were three to four orders of magnitude higher. From the different embryonic stages considered transporter transcript levels of both transporters were highest at $1 \mathrm{hpf}$; $a b c b 4$ was lowest at $12 \mathrm{hpf}$, increasing from there on; $a b c b 5$ levels varied from stage to stage (Table 1). Transcripts in 1 and 6 hpf embryos are maternally transferred mRNA as de novo transcription is initiated between mid-blastula (1 k-cell stage: $3 \mathrm{hpf}$ ) and gastrula stages (75\% epiboly; 7 hpf) [19].

Whole-mount in situ hybridization (WISH) revealed ubiquitous, weak constitutive $a b c b 4$ expression in the developmental period of zebrafish embryos examined here, which is indicated by faint purple staining of the entire embryos at the examined stages (Figure 2). Specificity of the probe we used was confirmed with $120 \mathrm{hpf}$ embryos where a strong WISH signal occurred in the gut (Additional file 1: Figure S3). When assuming that transcript levels are indicative of protein expression and activity, the ubiquitous expression of $a b c b 4$ transcripts found in 18 and 36 hpf embryos suggests a general continuous Abcb4 activity in all embryonic cells in this developmental phase.

Expression of $a b c b 5$ transcripts has been found in epidermal cells of zebrafish embryos [20], which could point to a similar function of the protein as in mammals where it regulates membrane potential and cell fusion of skin progenitor cells [21].

\section{Abcb4 but not Abcb5 antagonizes accumulation of fluorescent transporter substrates in zebrafish embryos} Having shown that $a b c b 4$ and $a b c b 5$ transcripts are present in zebrafish embryos, we examined the function of the corresponding proteins as efflux pumps. Rhodamine B and calcein-am served as proxies for efflux transporter activity. Efflux transporter activity is indicated when the accumulation of fluorescent dye substrates is increased in cells due to disrupted transporter activity and, hence, disrupted active efflux of dye by transporter inhibiting chemicals [22] or by knock-down of the transporter protein.

Table 1 Relative $a b c b 4$ and $a b c b 5$ mRNA abundances in zebrafish embryos at different developmental stages

\begin{tabular}{cccccc}
\hline hpf & 1 & 6 & 12 & 24 & 48 \\
\hline$a b c b 4$ & $57.09 \pm 13.03$ & $2.36 \pm 0.91$ & $0.91 \pm 0.26$ & $2.03 \pm 0.31$ & $11.62 \pm 0.99$ \\
$a b c b 5$ & $11.81 \pm 1.87$ & $2.05 \pm 0.74$ & $7.81 \pm 0.74$ & $2.36 \pm 1.19$ & $0.614 \pm 0.09$ \\
$\beta$-actin & $20,575 \pm 572$ & $15,613 \pm 6,938$ & $24,639 \pm 9,007$ & $17,740 \pm 3,948$ & $22,004 \pm 6,891$ \\
\hline
\end{tabular}

The mRNA abundances were determined in embryos at 1, 6, 12, 24 and 48 hours post fertilization (hpf). The expression levels that were normalized to 185 rRNA are shown as means \pm SD of four independent RNA isolations. Expression values were multiplied by $10^{6}$ for clarity of presentation. Beta-actin transcript levels are exemplarily shown for a highly expressed gene for comparison. 


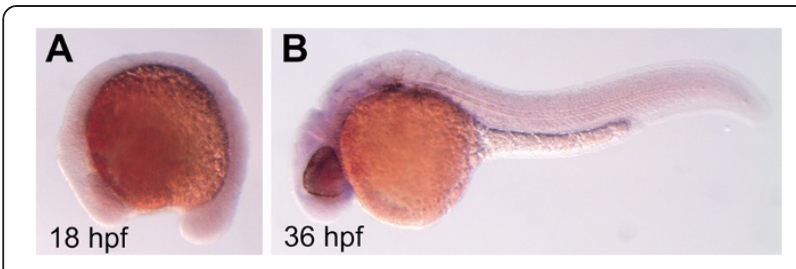

Figure 2 Images of $18 \mathrm{hpf}(\mathrm{A})$ and $36 \mathrm{hpf}(\mathrm{B})$ zebrafish embryos showing $a b c b 4$ expression patterns. Abcb4 transcripts where visualized with whole mount in situ hybridization. The faint but global staining patterns indicate weak, ubiquitous constitutive expression of abcb4 in the embryos at both stages.

\section{Effects of transporter inhibitors on accumulation of} rhodamine $B$ and calcein-am/calcein in tissues of zebrafish embryos

We measured changes in uptake of these dyes by embryos in the presence of two pharmacologic inhibitors of mammalian P-glycoproteins, namely cyclosporin A [23] and PSC833 [24], and of MK571, an inhibitor of mammalian ABCC transporters [25]. Fluorescence micrographs of embryos show that rhodamine B accumulated mainly in the yolk sac, whereas calcein fluorescence appeared in the head, trunk and cells scattered in the embryo body surface (Figure 3). Calcein-am is nonfluorescent, but once inside cells, it is hydrolyzed by cytosolic esterases and forms green, fluorescent calcein. Thus, calcein fluorescence in addition to efflux transporter activity also depends on the rate of calcein formation by esterases. Rhodamine $\mathrm{B}$, on the other hand, is already fluorescent without modification in the cell and these differences may explain the differing spatial accumulation patterns of the dyes in embryo tissues. Fluorescence intensities of both dyes were increased when cyclosporin A, PSC833 or MK571 (Figure 3A) was also present, indicating elevated dye accumulation in the embryos. Thus, without an inhibitor, the accumulation of dyes in the embryos is kept low, evidencing that both rhodamine B and calcein-am are kept out of embryo tissues by an active efflux mechanism.

For quantifying amounts of accumulated dye in embryos of different developmental stages, we measured rhodamine $\mathrm{B}$ levels in embryo tissue extracts following

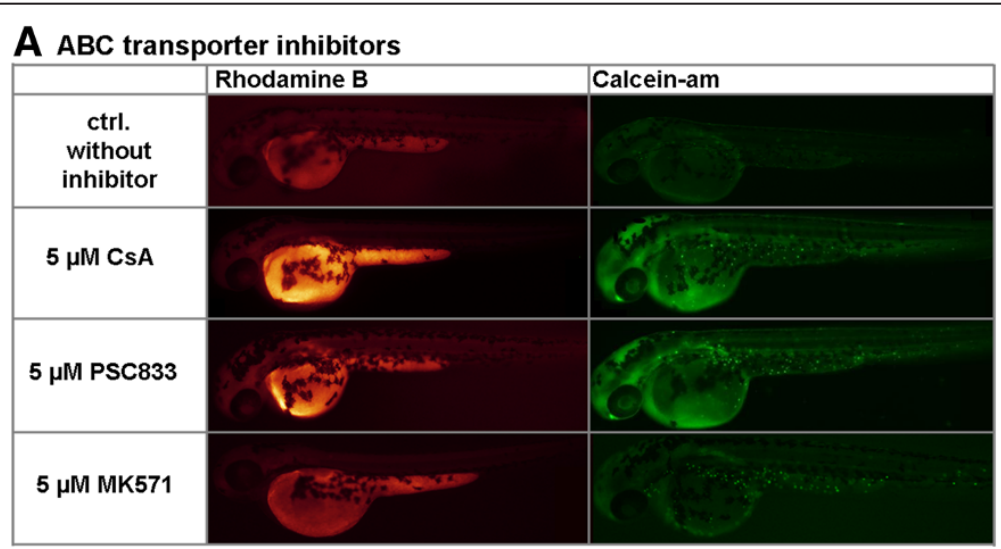

B Morpholino knock-down

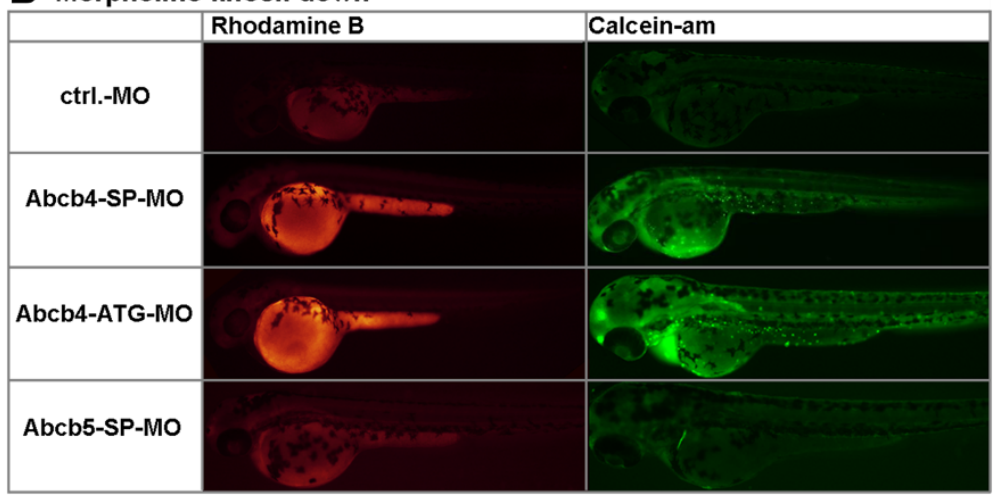

Figure 3 Fluorescence micrographs of $48 \mathrm{hpf}$ zebrafish embryos that had been incubated with $0.5 \mu \mathrm{M}$ rhodamine $\mathrm{B}$ or $1.0 \mu \mathrm{M}$ calceinam either in the presence of different $A B C$ transporter inhibitors (A) or upon knock-down of Abcb4 or Abcb5 (B). $A B C$ transporter inhibitor compounds were cyclosporin A (CSA), PSC833 and MK571. Morpholino oligonucelotides were used for knock-down of Abcb4 (Abcb4-SP$\mathrm{MO} / \mathrm{Abcb} 4-\mathrm{ATG}-\mathrm{MO}$ ) or Abcb5 (Abcb5-SP-MO). Exposure water of control embryos contained dye only (ctrl, ctrl-MO). The morpholino control (ctrl-MO) served to exclude unspecific morpholino effects on dye accumulation in the embryos. Brighter appearing embryos indicate accumulation of higher amounts of dye in the embryo tissue. 
one hour of dye exposure. Rhodamine B amounts per embryo in controls, indicating the basal accumulation of the dye in the embryos with efflux transporters functioning, were approximately $200 \mathrm{fmol}$ at $1 \mathrm{hpf}$, increasing to approximately $350 \mathrm{fmol}$ at $6 \mathrm{hpf}, 12 \mathrm{hpf}$ and at $24 \mathrm{hpf}$ and approximately $400 \mathrm{fmol}$ at $48 \mathrm{hpf}$ (Figure 4A, Additional file 1: Table S3). Enhanced accumulation of dye with the inhibitors cyclosporin A, PSC833 and
MK571 was seen in embryos of all tested developmental stages, which demonstrates efflux transporter activities in all those stages. Rhodamine B tissue levels were elevated with increasing inhibitor concentrations and were up to 1.8- to 3.25-fold higher compared to controls (Figures 4A, Additional file 1: Table S3). For example, at $10 \mu \mathrm{M}$ cyclosporin A or $10 \mu \mathrm{M}$ PSC833, amounts of rhodamine B per embryo at $48 \mathrm{hpf}$ were approximately
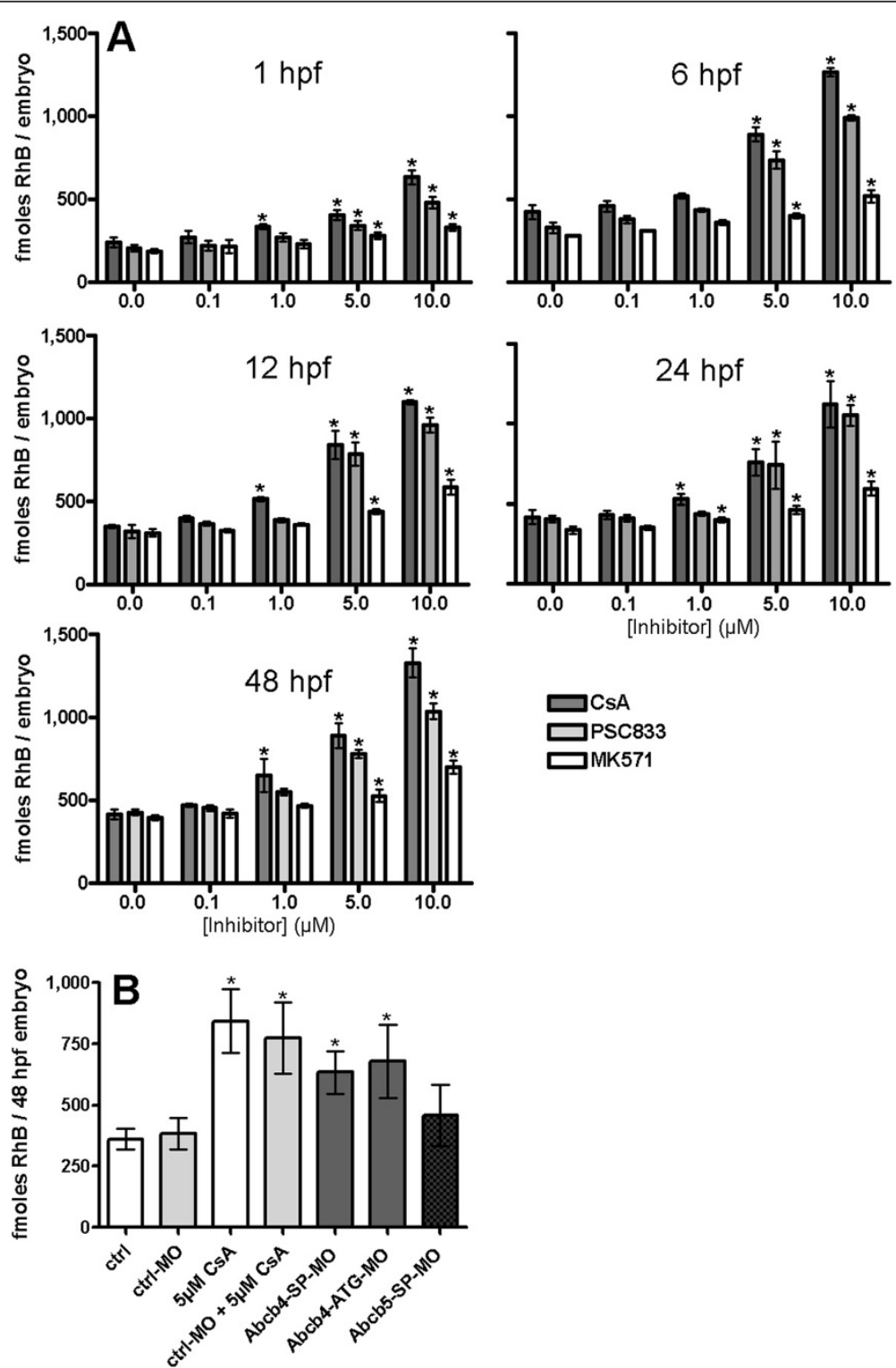

Figure 4 Quantification of rhodamine B (RhB) dye accumulation in zebrafish embryos. (A) Amounts of RhB accumulated in 1 hpf, 6 hpf, 12 hpf, $24 \mathrm{hpf}$ and $48 \mathrm{hpf}$ zebrafish embryos that, simultaneously to RhB, were exposed to various concentrations of transporter inhibitors cyclosporin A (CsA), PSC833 or MK571. (B) Amounts of RhB accumulated in 48 hpf zebrafish embryos upon morpholino knock-down of Abcb4 (Abcb4-SP-MO/Abcb4-ATG-MO) or Abcb5 (Abcb5-SP-MO). Controls contained dye only (ctrl, ctrl-MO), the morpholino control (ctrl-MO) served to exclude unspecific morpholino effects on dye accumulation in the embryos. Experiments with CsA (5 $\mu \mathrm{M}$ CsA, ctrl-MO + $5 \mu \mathrm{M}$ CsA) were set up as comparisons for the effects of this pharmacologic transporter inhibitor on RhB accumulation in the embryos. Values are means $+/-$ standard deviations from three independent experiments. Statistically significant differences from controls were determined with one-way analysis of variance (ANOVA), followed by Dunnett's test and are indicated by asterisks $(P<0.05)$. 
1,300 fmol and approximately 1,000 fmol, respectively. MK571 also increased dye accumulation in the embryos, but it was less potent than cyclosporin A and PSC833. Thus, at $10 \mu \mathrm{M}$ MK571, rhodamine B amounted to approximately $700 \mathrm{fmol}$ per $48 \mathrm{hpf}$ embryo.

\section{Effects of morpholino knock-down of Abcb4 and/or Abcb5 on accumulation of rhodamine $B$ and calcein-am/calcein in tissues of zebrafish embryos}

We examined whether Abcb4 and/or Abcb5 mediate cellular efflux activity in the embryos with morpholino knock-down. We used splice-blocking morpholinos for knock-down of Abcb4 (Abcb4-SP-MO, $0.5 \mathrm{mM}$ ) and Abcb5 (Abcb5-SP-MO, $0.5 \mathrm{mM}$ ) proteins, respectively, and assayed rhodamine $\mathrm{B}$ and calcein-am uptake by those embryos at $48 \mathrm{hpf}$. Control morpholino-treated embryos (Crtl-MO, $1 \mathrm{mM}$ ) served as the control for unspecific effects of morpholino injection on dye uptake. Abcb4 and Abcb5 knock-down did not cause phenotypic or necrotic effects, indicating that both Abcb4 and Abcb5 are not essential for development or basic physiological homeostasis in embryos up to $48 \mathrm{hpf}$. This is reminiscent of Abcb1 knock-out mice that also develop normally [3]. In the dye assays, rhodamine B and calcein fluorescence were brighter upon Abcb4 knock-down compared to the control, whereas Abcb5 knock-down had no visible effect on dye uptake (Figure 3B). Upon Abcb4 knock-down, approximately 650 fmol rhodamine $\mathrm{B}$ accumulated per $48 \mathrm{hpf}$ embryo compared to approximately $450 \mathrm{fmol}$ and approximately $400 \mathrm{fmol}$ in Abcb5 knock-down and control morpholino treated embryos, respectively (Figure 4B). Abcb4 knock-down with a translation-blocking morpholino (Abcb4-ATG$\mathrm{MO}, 0.0625 \mathrm{mM}$ ) resulted in accumulation of approximately 650 fmol rhodamine B per embryo, which is similar to the effect of the Abcb4 splice-blocking morpholino (Figures $3 \mathrm{~B}$ and $4 \mathrm{~B}$ ). These results show that changes in rhodamine $\mathrm{B}$ or calcein accumulation were specifically related to Abcb4 knock-down. Thus, Abcb4, but not Abcb5, appears to act as an efflux pump of rhodamine $B$ and calcein-am, respectively, keeping tissue concentrations of these compounds in zebrafish embryos low.

From these data, efflux of rhodamine B can clearly be associated with Abcb4 activity; however, the effect of Abcb4 knock-down on rhodamine $B$ accumulation in the embryos was not as pronounced as with high cyclosporin A and PSC833 concentrations (Figure 4A). The reason for this discrepancy of Abcb4 knock-down and inhibitor effects could be that morpholino knock-down does not result in complete suppression of Abcb4 protein function. Thus, morpholino treatment that leads to transient knock-down of correct pre-mRNA splicing or mRNA translation does not necessarily result in complete elimination of functional protein; thus, to a certain extent, functional Abcb4 may still have been present in Abcb4 knock-down embryos. Rhodamine B efflux could, apart from Abcb4, be mediated also by other transporters that remain functional in Abcb4 knock-down embryos, but are blocked by chemical inhibitors. There is, for example, indications that cyclosporin A and PSC833, apart from Abcb, also block activity of Abcc transporters of fish [26]. In addition to Abcb4, Abcc transporters that have earlier been shown to be expressed in zebrafish embryos [27] may play a role in rhodamine $\mathrm{B}$ efflux. However, our data indicate that the role of Abcc transporters in efflux of rhodamine $B$ is minor: MK571, an inhibitor of mammalian ABCCs, has a comparatively small effect on rhodamine $B$ efflux (Figures 3A and 4A). In addition, the MK571 effect on rhodamine $B$ accumulation may in fact be Abcb4 inhibition as is indicated by our tests with MK571 and recombinant Abcb4 (Figure 5E, see below). These results thus support that rhodamine B efflux in zebrafish embryos is majorly mediated by Abcb4 and that Abcb4 knock-down does not completely disrupt Abcb4 function.

\section{Effects of other chemicals on rhodamine B efflux in zebrafish embryos}

Galaxolide, tonalide, phenanthrene, verapamil and vinblastine also caused increased accumulation of rhodamine B in 48 hpf zebrafish embryos in a concentrationdependent manner (Figure 6, Additional file 1: Table S3). The data confirm interaction of those compounds with the rhodamine B efflux mechanism in zebrafish embryos that, as we show here, is Abcb4. Levels of rhodamine B in embryo tissue were significantly increased in zebrafish embryo tissue at concentrations $\geq 0.1 \mu \mathrm{M}$ tonalide, $\geq 1 \mu \mathrm{M}$ phenanthrene and galaxolide, and $\geq 2.5 \mu \mathrm{M}$ verapamil and vinblastine (Figure 6, Additional file 1: Table S3). Galaxolide, tonalide and phenanthrene, which were included in the tests as ecotoxicologically relevant chemicals, were as effective as the inhibitors cyclosporin A and PSC833 causing accumulation of approximately 1,100 to 1,500 fmol rhodamine B per embryo, equivalent to 2.4- to 2.9-fold increases compared to the controls. Galaxolide and tonalide, polycyclic musk compounds, have earlier been found to act as efflux transporter inhibiting chemosensitizers in marine bivalves [30]; the data in this study indicate that the musks also affect the efflux of rhodamine $B$ in zebrafish embryos, which appears to be conferred by Abcb4 (Figure 5J-K, see below). The effect of verapamil, a potent inhibitor of mammalian $A B C B 1$, was not as high as was found with the other ABCB1 inhibitors, cyclosporin A and PSC833. The amount of rhodamine B was maximally 860 fmol per embryo, corresponding to a 1.9- 


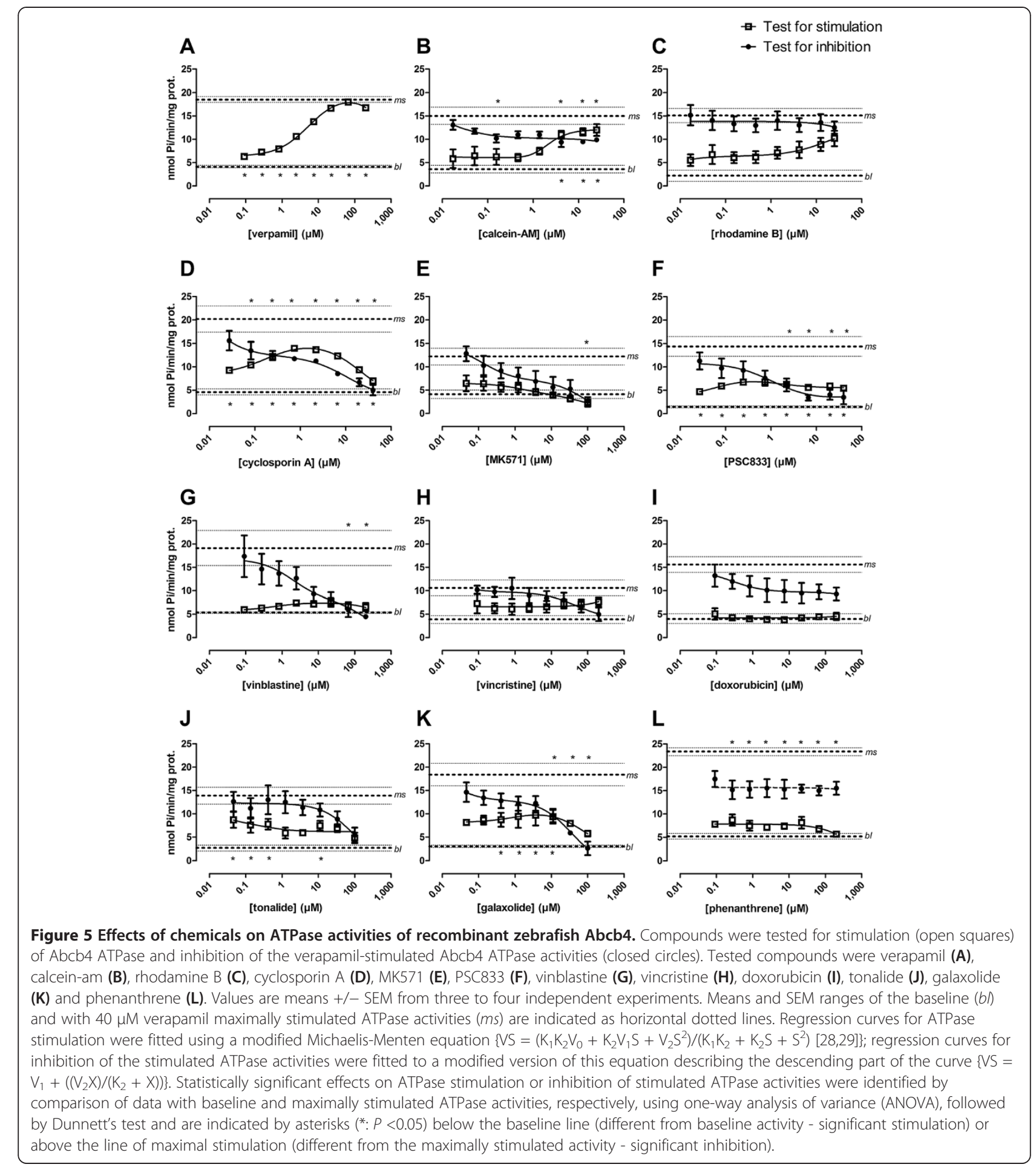

fold increase of rhodamine B accumulated in embryos with verapamil present (Figure 6, Additional file 1: Table S3). Interestingly, however, our assays with recombinant protein indicated strong interaction of verapamil with Abcb4 from zebrafish, suggesting that verapamil acts as a substrate of this transporter (Figure 5A, see below). Being a substrate of the efflux transporter, verapamil may cause competitive inhibition of the transporter function and, therefore, its inhibitory potency depends on the degree of interference with another substrate (in our case rhodamine B) when both bind to the substrate binding site of the protein. Thus, interference can be low if two compounds bind to different sites of the substrate binding site [31]. The discrepancy of 


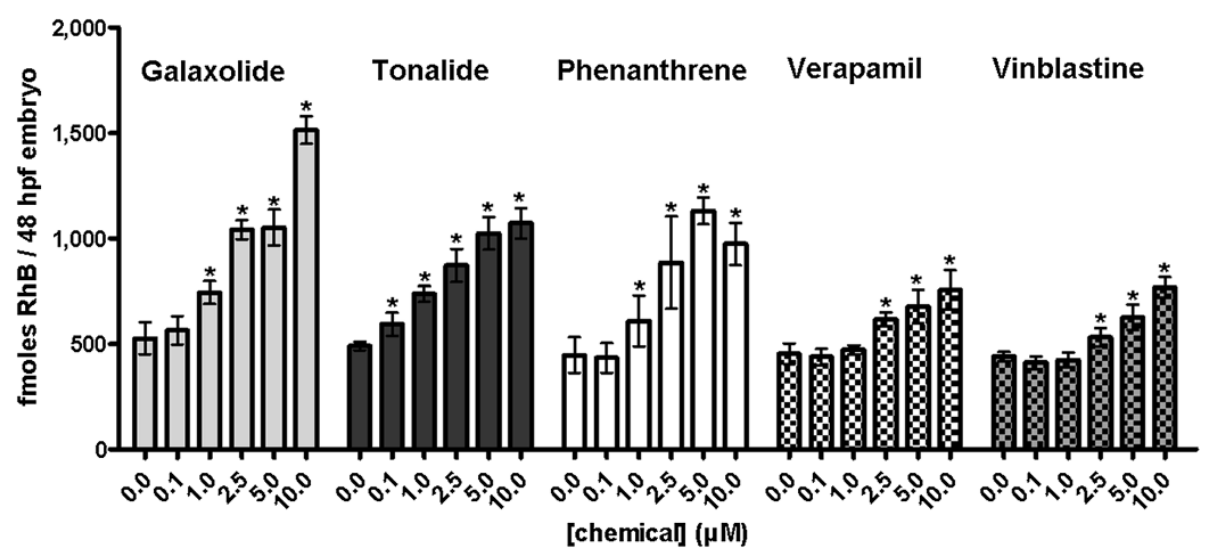

Figure 6 Amounts of rhodamine B (RhB) accumulated in $48 \mathrm{hpf}$ zebrafish embryos when also exposed to different concentrations of various chemicals. These compounds comprised the synthetic musk fragrances galaxolide and tonalide, the polycyclic aromatic hydrocarbon phenanthrene, the ABCB1 inhibitor verapamil and the ABCB1 substrate vinblastine. Galaxolide, tonalide and phenanthrene are ecotoxicologically relevant chemicals. Embryos were exposed to RhB and simultaneously to either of these compounds. Values are means $+/-$ standard deviations from three independent experiments. Statistically significant differences from controls were determined with one-way analysis of variance (ANOVA), followed by Dunnett's test and are indicated by asterisks $(P<0.05)$.

comparatively weak inhibition of rhodamine B efflux and strong interaction with the transporter protein ATPase by verapamil could thus be explained with little interference of the compounds when binding to the Abcb4 binding site. All of the tested compounds interacted with recombinant Abcb4 in the ATPase assay (Figure 5; see below) suggesting that their effect on rhodamine $\mathrm{B}$ accumulation in the embryos is associated with inhibition of the Abcb4-mediated efflux activity of rhodamine $\mathrm{B}$.

Disruption of Abcb4 activity leads to increased sensitivity of embryos to toxic transporter substrates

Effects of $A B C$ transporter inhibitors on the mortality of embryos due to toxic compounds

We chose vinblastine, vincristine and doxorubicin, cytotoxic substrates of human ABCB1 [32-34], and phenanthrene as an ecotoxicologically relevant model compound, for determining to what extent chemical resistance of zebrafish embryos is associated with $\mathrm{ABC}$ transporter efflux activity. In initial experiments that served to determine concentrations of the compounds that were toxic to zebrafish embryos we focused on the micromolar concentration range in which interaction of chemicals with transporters is generally observed. When embryos were exposed to the compounds from 1 to $48 \mathrm{hpf}$, we found lethal effects of vinblastine at concentrations $>1 \mu \mathrm{M}$ and $100 \%$ mortality at concentrations $\geq 5 \mu \mathrm{M}$; of vincristine at concentrations $\geq 10 \mu \mathrm{M}$; and of phenanthrene at concentrations $>1 \mu \mathrm{M}$ and $100 \%$ mortality at concentrations $\geq 20 \mu \mathrm{M}$. Within the concentration range tested (up to $40 \mu \mathrm{M}$ ), vincristine did not cause $100 \%$ mortality. Toxicity of doxorubicin for zebrafish embryos appears to be low; we found no toxic effects for the compound at concentrations in the micromolar range and, indeed, lethal effects were reported for substantially higher concentrations [35].

In further experiments that served to explore the role of transporter activity for the sensitivity of zebrafish embryos to toxic compounds, the test compounds were applied in a concentration series with two concentrations of vincristine $(20,40 \mu \mathrm{M})$ that were found to be toxic and for vinblastine and phenanthrene within the range causing up to $100 \%$ mortality in zebrafish embryos; doxorubicin was not further considered in these experiments. Toxicities of vinblastine, vincristine and phenanthrene were compared when applied alone and in combination with the non-toxic concentration of $5 \mu \mathrm{M}$ of the transporter inhibitor cyclosporin $\mathrm{A}$ and, in the case of vinblastine, as well with $5 \mu \mathrm{M}$ PSC833.

In an experimental series with vinblastine and cyclosporin A, LC50 values (concentration that was lethal for $50 \%$ of embryos) for vinblastine after exposure from 1 to $48 \mathrm{hpf}$ were $3.05 \mu \mathrm{M}$ (95\% CI (confidence interval): 2.94 to $3.17 \mu \mathrm{M})$ without and $2.37 \mu \mathrm{M}(95 \% \mathrm{CI}: 2.25$ to $2.49 \mu \mathrm{M}$ ) with cyclosporin A (Figure 7A), which is a difference of $22.3 \%$ (Additional file 1: Table S4). A similar decrease in LC50 for vinblastine was seen with PSC833 (Figure 7B) indicating higher toxicity of vinblastine when the transporter inhibitors were present. For testing whether the increase in vinblastine toxicity was indeed due to higher accumulations of the compound in the embryos, we studied uptake of bodipylabeled, fluorescent vinblastine by embryos. Fluorescence intensities were elevated in embryos treated with $1.0 \mu \mathrm{M}$ bodipy-vinblastine combined with cyclosporin A compared to embryos exposed to bodipy-vinblastine only (Figure 8A). This indicates that accumulation of 


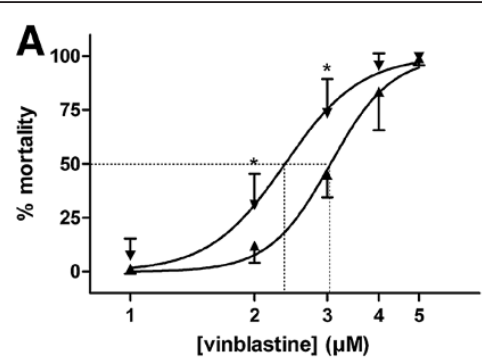

$\Delta$ without CsA

v with $5 \mu \mathrm{M}$ CsA

B

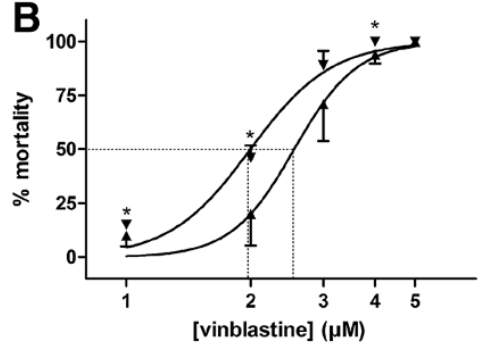

- without PSC833

vith $5 \mu \mathrm{M}$ PSC833
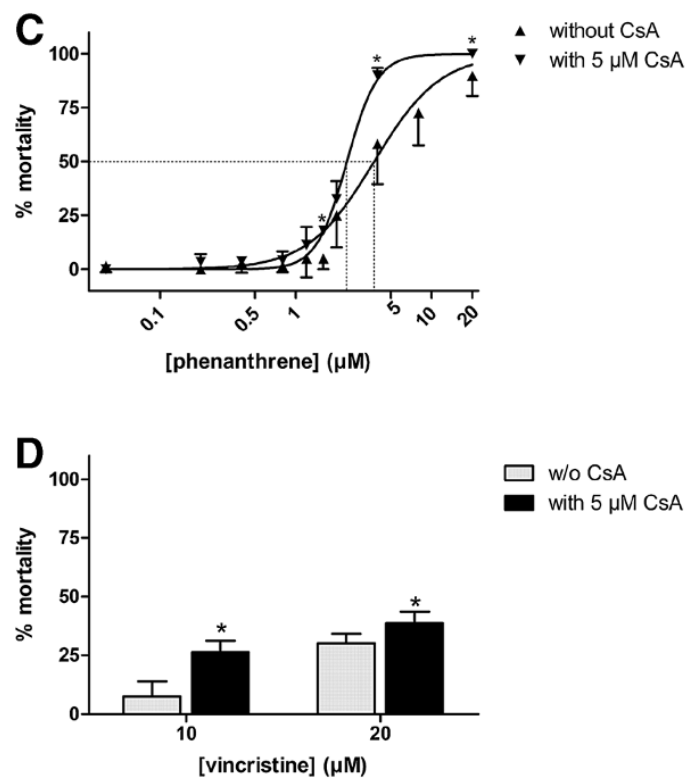

Figure 7 Percent mortalities of zebrafish embryos after 1 to 48 hpf exposures to toxic compounds in the presence or absence of $A B C$ transporter inhibitors. The graphs show mortalities caused by different concentrations of vinblastine without and with $5 \mu \mathrm{M}$ CSA (A) or $5 \mu \mathrm{M}$ PSC833 (B) and of phenanthrene (C) and of vincristine (D) without and with $5 \mu \mathrm{M}$ CsA. Depicted are mean values and standard deviations of independent replicates. For vinblastine and phenanthrene concentration-response regressions were determined using the logistic HILL model with bottom restrained to 0 and top restrained to 100 . The dotted lines indicate the concentration causing mortality in $50 \%$ of the embryos (LC50). Refer to Additional file 1: Table S3 for details on the number of independent replicates, LC50 values with confidence intervals and further curve parameters. Statistically significant differences in mortalities between treatments without and with inhibitors at respective vinblastine, vincristine or phenanthrene concentrations were determined with the paired (vinblastine, vincristine) or the regular t-test (phenanthrene) and are indicated by asterisks $(P<0.05)$. bodipy-vinblastine in the embryos depends on efflux transporter activity and confirms that higher vinblastine toxicity in the presence of inhibitors is due to reduced efflux and thus increased accumulation of the compound in the embryos.

As found for vinblastine, mortalities of embryos were significantly increased when vincristine and phenanthrene, respectively, were combined with $5 \mu \mathrm{M}$ cyclosporin A, likewise indicating higher accumulation of those compounds in the embryos when cellular efflux activity is blocked (Figures 7C, D). Mortalities caused by 10 and 20 $\mu \mathrm{M}$ vincristine were increased by 3.5 - and 1.3-fold when cyclosporine was also present (Figure 7D). Cyclosporin A had a significant effect on the toxicity of phenanthrene, evidenced by a $37 \%$ difference between the LC50 values for phenanthrene alone $(3.8 \mu \mathrm{M})$ and with cyclosporine $(2.4 \mu \mathrm{M})$ (Figure 7C, Additional file 1: Table S3).

\section{Effects of morpholino knock-down of Abcb4 or Abcb5 on vinblastine-caused mortalities in embryos}

We furthermore examined the effect of disruption of transporter activity on resistance of embryos to vinblastine using morpholino knock-down. After 48 hours, exposure to $2 \mu \mathrm{M}$ vinblastine caused $74 \% \pm 10.2$ mortality in embryos upon knock-down of Abcb4, whereas Abcb5 knock-down and control morpholino embryos showed mortalities ( $53 \% \pm 10.6$ and $50 \% \pm 12.3$, respectively) similar to untreated controls (ctrl; 44\% \pm 13.2 ) (Figure 8B). As with the Abcb4 splice-blocking morpholino treated embryos, elevated sensitivity to vinblastine was also found for embryos treated with the Abcb4 translation-blocking morpholino (Figure 8B). The experiments with bodipyvinblastine support the interpretation that stronger effects by vinblastine upon Abcb4 knock-down are associated with increased vinblastine accumulation in the embryos. Thus, as with cyclosporin A, the bodipy-vinblastine fluorescence in embryos was likewise increased when Abcb4 knock-down embryos (Figure 8A). When non-morpholino, control morpholino or Abcb5 knock-down embryos were co-treated with cyclosporin A or PSC833, mortalities by $2 \mu \mathrm{M}$ vinblastine were raised to similar levels as those in embryos with Abcb4 knock-down without an inhibitor. The similar effects of Abcb4 knock-down and of transporter inhibitor compounds indicate that Abcb4 has a major role in mitigating vinblastine toxicity in zebrafish embryos and that the sensitivity-enhancing effect of the inhibitors, cyclosporin A and PSC833, is blocking activity of this transporter. The effect in treatments with Abcb4 knock-down and inhibitors in combination tended to be slightly stronger than in treatments with only Abcb4 knock-down or inhibitors (Figure 8B). This can be seen as an indication that neither Abcb4 knock-down nor treatment with the inhibitors at the concentration applied lead to complete suppression of Abcb4 function, which is in 


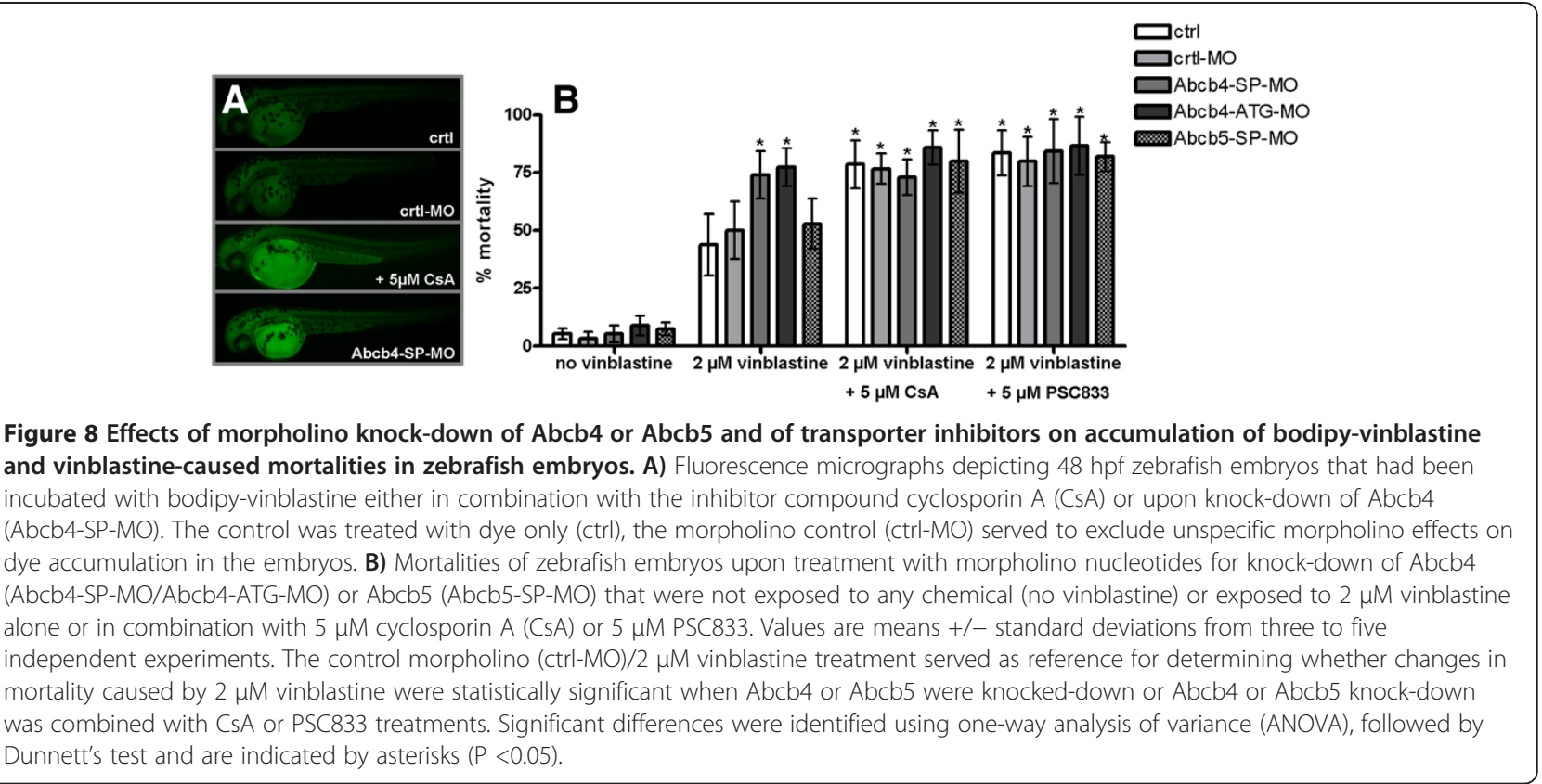

concordance with the findings in the rhodamine dye accumulation experiments (see above, Figure 4B).

\section{Abcb4 substrates and inhibitors modulate the ATPase activity of recombinant zebrafish Abcb4}

Our results from the dye uptake and toxicological assays indicate that (1) zebrafish embryos employ a multidrug transporter like cellular efflux mechanism that blocks uptake of chemicals by the embryos from the water and thus mitigates their toxic effects; (2) this cellular efflux of compounds is largely based on the activity of Abcb4, but not of Abcb5; and (3) effects of transporter inhibitors are associated with inhibition of Abcb4 activity.

Further evidence for interaction of the tested compounds with Abcb4 as substrates and/or inhibitors comes from assays where effects of chemicals on the ATPase activity of recombinant Abcb4 protein were quantified. As a measure for interaction of chemicals with Abcb4, changes in ATPase activity of recombinant Abcb4 with the tested chemicals present were used (Figure 5). Stimulation of the basal transporter ATPase activity, indicated as the amount of inorganic phosphate $(\mathrm{Pi})$ released during the hydrolysis of ATP, occurs with compounds acting as substrates; inhibition of the stimulated ATPase activity is found for transporter inhibitors [36]. For stimulation of Abcb4 in the inhibition experiment and as a positive control we used verapamil, which stimulated the Abcb4 ATPase activity by 4.4-fold (mean of positive controls from all experiments). The action of verapamil on zebrafish Abcb4 is comparable to its effect on the human ABCB1 ATPase activity, which is stimulated by 4.5 - to 5.0-fold [36,37].

\section{Stimulation of the basal Abcb4 ATPase activity}

The compounds that were applied as fluorescent (rhodamine $B$, calcein-am) or toxic (vinblastine, vincristine, phenanthrene) transporter substrates in experiments with the zebrafish embryos generally stimulated the basic Abcb4 ATPase activity (Figure 5B, C, G, H, L) although the effect was statistically significant only for calcein-am (Figure 5B). However, the Pi levels were generally above baseline also with rhodamine $\mathrm{B}$, vinblastine, vincristine and phenanthrene (Figure 5C, G, H, L), which can be seen as an indication that these compounds also stimulated the Abcb4 ATPase activity, albeit to a lesser extent. Doxorubicin stimulates the ABCB1 ATPase activity only at very high concentrations [37] and, accordingly, the compound had no effect on the zebrafish Abcb4 ATPase activity in the concentration range we tested (Figure $5 \mathrm{I}$ ).

Apart from the classic ABCB1 inhibitor verapamil, the other tested inhibitors, cyclosporin A and PSC833, significantly and, in the case of MK571, weakly, stimulated the Abcb4 ATPase activity (Figure 5D-F). The ATPase stimulating effect of cyclosporin A is consistent with its property as substrate of the mammalian ABCB1 [38], even though, in contrast to our results with $\mathrm{Abcb} 4$, the compound does not stimulate the ABCB1 ATPase [37]. Interestingly, substantial Abcb4 ATPase stimulation was seen with tonalide and galaxolide, indicating their properties as Abcb4 substrates (Figure 5J, K). However, as the compounds are very lipophilic [39], they probably highly accumulate in the cellular membranes and it is, therefore, questionable if Abcb4 activity leads to efflux of the compounds from the cells. 
Activation of Abcb4 ATPase appeared in the nano- to low micro-molar concentration range of compounds corresponding to the concentrations applied in the dye uptake and toxicological assays with zebrafish embryos. This implies that these concentrations were in a range where the compounds are actively transported by Abcb4.

In the case of cyclosporin $\mathrm{A}$, the concentrationdependent Abcb4 ATPase activity showed an initial increase in activity, followed by an activity decrease at higher concentrations (Figure 5D). This biphasic response in ATPase activities to increasing concentrations of a compound is common for substrates of human $\mathrm{ABCB} 1$ and it is associated with the presence of two binding sites with different binding affinities in the protein, one for stimulation (with a high affinity that is activated at low concentrations) and one for inhibition (with a low affinity that is activated at higher concentrations) [28]. A decrease in ATPase stimulation at higher concentrations was observed as a trend also for MK571, vinblastine, galaxolide, tonalide and phenanthrene (Figure 5E, G, J, K, L).

\section{Inhibition of the stimulated Abcb4 ATPase activity}

Except for rhodamine B (Figure $5 \mathrm{C}$ ), all of the tested compounds caused a decrease in the stimulated Abcb4 ATPase activity and the results are congruent with the sensitizing effects of cyclosporin A and PSC833 in the embryotoxicity tests (Figures 7 and 8) and with the effects of cyclosporine A, PSC833, MK571, vinblastine, tonalide, galaxolide and phenanthrene on rhodamine $\mathrm{B}$ accumulation in embryo tissue (Figures $3 \mathrm{~A}, 4$ and 6). For MK571, which is an inhibitor of mammalian $\mathrm{ABCC} 1$, the inhibiting effect indicates that it also acts on zebrafish Abcb4 and, as discussed above, its effects on rhodamine $B$ accumulation in the zebrafish embryos may in fact be from inhibition of Abcb4 in the embryos.

\section{Conclusions}

We here show that cellular efflux activity of an Abcb4 ortholog in zebrafish embryos in the first 48 hours of development antagonizes uptake of chemicals from the water. This is evidenced by the finding that the presence of $\mathrm{ABC}$ transporter inhibitors as well as morpholino knock-down of expression of functional Abcb4 leads to increased accumulation of toxic and fluorescent transporter substrates in the embryos. ATPase assays with recombinant zebrafish Abcb4 confirm the properties of our test compounds as Abcb4 substrates and/or inhibitors. Since morpholino knock-down of Abcb4 and exposure of embryos to chemical transporter inhibitors had similar effects on accumulation of substrates in the embryos it can be concluded that zebrafish Abcb4 is a major component of the MXR system of zebrafish embryos.

This multixenobiotic resistance mediating function of the protein contrasts to mammalian $\mathrm{ABCB} 4$, which is a specialized translocator of phosphatidylcholine (PC) into bile that transports cytotoxic drugs only at low rates and does not confer multixenobiotic resistance [8]. Contrary to this, Abcb4 in fish may not act as a PC translocator as is indicated by the lack of PC in the bile of the Asian carp [40], which, like zebrafish, is a cyprinid. The ability to transport a wide range of toxicants is probably a primary, ancient property of P-glycoproteins that is conserved for Abcb4 in fish. This could explain why fish tolerate the absence of Abcb1 since function as a toxicant pump is executed by the Abcb4 ortholog. In adult zebrafish, Abcb1-like efflux activity has been found to be a component of the blood-brain barrier [41]. As Abcb1 is absent in zebrafish, this efflux activity may well be associated with the function of the Abcb4 ortholog.

Abcb5, the other P-glycoprotein in zebrafish, appears not to mediate xenobiotic resistance.

The Abcb4-mediated efflux activity of toxicants in zebrafish embryos, a major pharmacologic and toxicologic model system, has important implications: 1) Abcb4 activity can substantially determine bioavailability - and thus pharmacologic and toxicologic potency - of a diverse array of chemicals to zebrafish embryos; 2) chemicals that modulate Abcb4 activity, such as the chemical transporter inhibitors we used, will enhance bioavailability of chemicals to zebrafish embryos that are usually effluxed by Abcb4. This chemosensitization by efflux transporter inhibition can be caused by a variety of chemicals, including seemingly innocuous ones [42]. The polycyclic musk compounds galaxolide and tonalide are an example for such compounds as they do not exert obvious toxic effects on zebrafish embryo development [43], but they clearly act as potent Abcb4 inhibitors. Our data underscore that efflux transporters are underappreciated but are important determinants of bioavailability of chemicals to cells and organisms. On the other hand, chemicals can reverse transporter function and thus increase bioavailability of other compounds. Depending on efflux transporter interference with different compounds, bioavailability of compounds in a mixture can deviate from that of the same components when they are present alone. This suggests that current regulatory practice of assessing risks based on individual compounds may underestimate toxicity. In this context, our baculovirus-based Abcb4 activity assay has proven reliable to determine substrate/inhibitor properties of test compounds and will be useful in chemical screens using zebrafish.

\section{Methods}

\section{Chemicals}

Cyclosporin A, doxorubicin hydrochloride, phenanthrene, rhodamine B, verapamil hydrochloride and vincristine sulfate were from Sigma-Aldrich (Schnelldorf, Germany). Calcein-acetoxymethylester (calcein-am), MK571 and vinblastine sulfate were from Biozol (Eiching, Germany) 
and bodipy-vinblastine was from Invitrogen (Karlsruhe, Germany). PSC833 was a kind gift from Novartis (Basel, Switzerland). Galaxolide (73\% of the total are diasterimeric isomers of 1,3,4,6,7,8-hexahydro-4,6,6,7,8,8-hexamethylcyclopenta- $\gamma$-[2]-benzopyran) was a kind gift from International Flavors \& Fragrances Inc. (IFF; Union Beach, NJ, USA) and Tonalide (7-acetyl-1,1,3,4,4,6-hexamethyl1,2,3,4-tetrahydronaphthalene; purity: 98\%) was obtained from Bush Boake Allen Inc. (Jacksonville, FL, USA). RhB was dissolved in MilliQ water; stock solutions of all other chemicals were prepared in dimethyl sulfoxide (DMSO, Sigma-Aldrich). Final DMSO solutions in exposure media did not exceed $0.2 \%$.

\section{Culture of zebrafish, collection of eggs and culture of embryos}

Adult zebrafish from the WIK wildtype strain were maintained and bred according to standard protocols [44]. Collection of eggs and culturing of the embryos were performed as described [45].

\section{RNA extraction and reverse transcription}

Total RNA was extracted from 30 to 50 embryos from 1, 6, 12, 24 and 48 hpf zebrafish embryos using TRIzol Reagent (Invitrogen) according to the manufacturer's instructions. Genomic DNA contaminations were removed with DNAse I (Roche, Grenzach, Germany) treatment. cDNA was synthesized from total RNA using the High Capacity cDNA Reverse Transcription Kit (Applied Biosystems, Darmstadt, Germany).

\section{Cloning of zebrafish $a b c b 4$ and $a b c b 5$ cDNAs and phylogenetic and synteny analysis}

Zebrafish abcb4 (predicted sequence accession no. ENSDARG00000010936) and $a b c b 5$ (predicted ENSDA RG00000021787) sequences were obtained using reverse transcription polymerase chain reaction (RT-PCR) with primer pairs designed either based on the predicted sequences or based on sequences obtained with rapid amplification of cDNA ends (RACE) (Clontech, Palo Alto, USA). For PCR, Advantage 2 (Clontech) and Phusion (Finnzymes, Thermofisher, Schwerte, Germany) polymerases were used. PCR products were gel purified, cloned and sequenced on an ABI 3100 sequencer (Applied Biosystems) using standard cycle sequencing protocols. Sequences were edited and assembled using Sequencher 4.9 (Genecodes, Ann Arbor, USA) and analyzed using the National Center for Biotechnology Information (NCBI) basic alignment search tool (BLAST) and the Expert Protein Analysis System (ExPASy, Swiss Institute of Bioinformatics, Lausanne, Switzerland) [46]. The zebrafish $a b c b 4$ and $a b c b 5$ sequences were submitted to GenBank (NCBI). Accession numbers are listed in Additional file 1: Table S2.

Identity rates of zebrafish transporter nucleotide/amino acid sequences with vertebrate orthologs were determined with ClustalX2 (Conway Institute UCD, Dublin, Ireland). Phylogentic trees were generated with MEGA5 (Center for Evolutionary Medicine and Informatics, Tempe, USA) using the neighbor-joining method with percentage concordance based on 1,000 bootstrap iterations. To establish syntenic relationships between vertebrate genomes within the chromosomal regions of interest, we made use of ortholog predictions in the Ensembl database [18].

\section{Quantification of mRNA expression levels in zebrafish embryos}

mRNA expression levels of $a b c b 4$ and $a b c b 5$ in 1, 6, 12, 24 and 48 hpf zebrafish embryos were quantified with quantitative RT-PCR (qPCR) using the SYBR Green PCR Master Mix (Quantace, Berlin, Germany). with an iCycler RealTime PCR Detection System (BioRad, Munich, Germany).

Primers for housekeeping and zebrafish ABC transporter genes (Additional file 1: Table S5) were designed against available mRNA sequences from Ensembl [18] and self-obtained sequences using Beacon Designer (Primer Biosoft, Palo Alto, USA). Samples were run in triplicate in optically clear 96-well plates (Biozym, Hessisch Oldendorf, Germany). PCR was performed with RNA extracts from three different zebrafish embryo batches. qPCR results were calculated relative to the housekeeping gene, $18 S$ (for selection of the housekeeping gene refer to Additional file 1: Figure S2), according to the normalization procedure of the Q-Gene Core Module [47-49], which takes varying PCR amplification efficiencies into account (Additional file 1: Table S6). All qPCR experiments were performed according to the MIQE (Minimum Information for Publication of Quantitative Real-Time PCR Experiments) guidelines [50]. A MIQE checklist is found in Additional file 1.

\section{Whole-mount in situ hybridization}

For whole-mount in situ hybridization (WISH), abcb4 cDNA fragments were amplified (for primers refer to Additional file 1: Table S7), cloned into pCRII (Invitrogen) and verified by sequencing. WISH with 18,38 and $120 \mathrm{hpf}$ (functional proof of the used probes, specific staining in the intestine, see Additional file 1: Figure S3) zebrafish embryos was performed as described previously [51]. WISH staining was analyzed with a stereomicroscope (MZ16F, Leica, Wetzlar, Germany).

\section{Procedure for measuring efflux transporter protein activity in zebrafish embryos with fluorescent dyes} Fluorescent dyes, rhodamine B [22,52], calcein-am $[53,54]$ and bodipy-vinblastine [55], served as proxies for efflux transporter activity in the fish embryos. When this activity is absent due to pharmacologic transporter inhibition or the absence of functional protein due to morpholino knock-down, accumulation of dye in the 
embryo tissue increases, resulting in a stronger fluorescence signal. Solutions for exposures were prepared in zebrafish embryo culture water with $0.5 \mu \mathrm{M}$ rhodamine $\mathrm{B}$, $1 \mu \mathrm{M}$ calcein-am and $1 \mu \mathrm{M}$ bodipy-vinblastine and inhibitors cyclosporin A (CsA), PSC833 and MK571, respectively. $\mathrm{Up}$ to 10 embryos per $\mathrm{mL}$ were incubated in the test solutions for one hour at $26^{\circ} \mathrm{C}$ in the dark, rinsed three times with clean culture water to remove dye from the chorion and subsequently photographed with a fluorescence microscope DMI 4000B (Leica) and DFC 350 FX camera (Leica). For quantification of RhB dye uptake, 10 embryos per treatment were sonicated in $200 \mu \mathrm{L}$ of a hypotonic lysis buffer (10 mM KCl, $1.5 \mathrm{mM} \mathrm{MgCl}_{2}, 10 \mathrm{mM}$ Tris $\mathrm{HCl}, \mathrm{pH} 7.4$ ), the sonicates were briefly centrifuged, $150 \mu \mathrm{L}$ of the supernatant were transferred to a black 96-well microplate (Nunc, Sigma-Aldrich, Schnelldorf, Germany) and the rhodamine B fluorescence was measured at $595 \mathrm{~nm}$ (emission)/530 nm (excitation) in a GENios plus fluorescence plate reader (Tecan, Männedorf, Switzerland). This assay enabled parallel examination of multiple treatments. Triplicates of five treatments along with a solvent control were run per experiment. Each experiment was repeated with embryos from three different egg batches laid on different days. The amount of rhodamine B accumulated in zebrafish embryos was quantified with a rhodamine B standard curve (Additional file 1: Figure S4).

\section{Embryo toxicity experiments}

For determining toxicities of vinblastine, vincristine and doxorubicin, 20 embryos were incubated in glass petri dishes with $10 \mathrm{~mL}$ test solutions and two to three replicates per treatment. Exposures to phenanthrene were set up in tightly closed glass vials containing $2 \mathrm{~mL}$ solution with four embryos per vial according to Schreiber et al. [56] to prevent volatilization of phenanthrene from the test solutions. Per tested treatment, five vials were set up in parallel. Exposures were started with 4- to 16-cell stage embryos to assure successful fertilization and terminated after 48 hours. Exposure experiments were repeated with at least three batches of embryos from different days. During exposure, embryos were regularly examined using a stereo microscope and dead embryos were removed and recorded. A final mortality count was performed at 48 hours and embryos were declared as dead if at least one of the following criteria applied: i) coagulation of eggs, ii) no heart beat, iii) no blood circulation, iv) no somites, v) tail not detached [45]. Initially, toxic concentration ranges of vinblastine, vincristine, doxorubicin and phenanthrene and the transporter inhibitors CsA and PSC833 were identified. Subsequently, effects of transporter inhibitors at non-toxic concentrations and, in the case of vinblastine, of morpholino knock-down of Abcb4 and Abcb5 on the sensitivity of the embryos to toxic test compounds were determined.
Controls contained i) $0.2 \%$ DMSO used as solvent, ii) inhibitors only or iii) morpholino knock-down embryos only. Mean mortality percentages and standard deviations at $48 \mathrm{hpf}$ were calculated from all experimental replicates and the paired $t$-test was applied to determine whether inhibitors or morpholino knock-down significantly modulated vinblastine sensitivity of embryos.

\section{Production of recombinant zebrafish Abcb4 protein with the baculovirus expression system and ATPase activity measurements}

The zebrafish abcb4 cDNA was sub-cloned into pFastBac1 (Invitrogen) and sequenced for confirmation. Abcb4 baculovirus was generated using the Bac-to-Bac Baculovirus Expression System (Invitrogen). Sf9 cells cultured in Sf-900 II SFM (Invitrogen) were used for virus amplification and protein expression. Crude Sf9 membranes with Abcb4 protein were prepared 60 hours after infection according to [57] and then stored at $-80^{\circ} \mathrm{C}$ until use. Total protein in the membrane preparations was quantified using the bicinchoninic acid assay (BCA) and bovine serum albumin (BSA) as standard and the presence of Abcb4 protein in the membranes was checked in $1 \mu \mathrm{g}$ of total protein by Western blotting using the anti-MDR1 antibody C219 (Additional file 1: Figure S5) as described previously [58].

ATPase assays were performed as described in [37] with minor modifications. Instead of $37^{\circ} \mathrm{C}$, incubations of the membranes with the test compounds were performed for 40 minutes at $27^{\circ} \mathrm{C}$, which is within the physiological temperature range of zebrafish. A total of $20 \mu \mathrm{g}$ of protein was used for each reaction. The ATPase stimulating effect of verapamil, a classical stimulating agent of the mammalian Abcb1 ATPase, was also detected for zebrafish Abcb4 (Figures 5A) and $40 \mu \mathrm{M}$ verapamil were used as positive control in ATPase stimulation assays and as ATPase stimulating agent in the ATPase inhibition assays. DMSO was used as the solvent for all compounds. The final DMSO concentration in the reaction was $2 \%$, which did not affect ATPase activities.

\section{Abcb4 and Abcb5 knock-down by morpholino (MO) microinjection}

Embryos were injected (FemtoJet, Eppendorf, Hauppauge, USA) through the chorion into the yolk compartment at the two-cell stage. Injection needles were pulled from borosilicate glass capillary tubes with filament (Warner Instruments, Hamden, USA) using a micropipette puller (Narishige, Tokyo, Japan).

Morpholinos (Gene Tools, Philomath, USA) were dissolved in MilliQ water and injected at the following concentration ranges: $0.5 \mathrm{mM}$ to $2 \mathrm{mM}$ Abcb4-MO-SP $(\mathrm{SP}=$ splice-blocking) (5'-AAT CTA ACT GCA TGA CGT ACT CTG T-3'); $0.0625 \mathrm{mM}$ to $1 \mathrm{mM}$ Abcb4-ATG-MO (translation-blocking) (5'-GCA AAC ATG GGC AAG AAA 
TCC AAA C-3'), $0.5 \mathrm{mM}$ to $2 \mathrm{mM}$ Abcb5-MO-SP (5'GCA ACA GGT ACA TTC ATG TCT TTC T-3') and 1 $\mathrm{mM}$ to $2 \mathrm{mM}$ control splice morpholino (ctrl-MO) (MO against human beta-globin) (5'- CCT CTT ACC TCA GTT ACA ATT TAT A-3'). Functionality of Abcb4-SP-MO and Abcb5-SP-MO morpholinos were proven by means of RT-PCR (Additional file 1: Figure S6) and Abcb4ATG-MO morpholino with co-injected Abcb4-ATG-GFP mRNA (Additional file 1: Figure S6). Injected embryos were cultured for $\leq 48 \mathrm{~h}$ and transporter activity dye assays and toxicity experiments were done as described above.

\section{Endnotes}

${ }^{a}$ Our designation of gene and protein names is based on the Zebrafish Nomenclature Guidelines [59]; fish: shh/ Shh, human: SHH/SHH, mouse: Shh/SHH (gene/protein).

\section{Additional file}

\section{Additional file 1: Additional information regarding the $\mathrm{qPCR}$} analysis procedure: MIQE (Minimum Information for Publication of Quantitative Real-Time PCR Experiments) checklist. Table S1 with percent similarity data from sequence comparisons. The table is supplementary to Figure 1B. Table $\mathbf{S} 2$ with the accession nos. of sequences used for phylogenetic analyses. The table is supplementary to Figure 1B. Table S3 with RhB amounts that had accumulated in 1, 6, 12, 24 and 48 hpf zebrafish embryos upon co-exposure to various compounds. The table is supplementary to Figures 4 and 6 . Table S4 with values from concentration-effect curves determined in zebrafish embryo toxicity experiments. The table is supplementary to Figure 7. Table S5 with sequences of primers used for $\mathrm{PPCR}$. The table is supplementary to Table 1. Table $\mathbf{S 6}$ with efficiencies of zebrafish abcb4, $a b c b 5$ and housekeeping primers used in GPCR reactions. The table is supplementary to Table 1. Table S7 with primer pairs used for PCR of zebrafish abcb4 fragments used for generating probes for whole-mount in situ hybridization (WISH). The table is supplementary to Figure 2. Figure S1 with conserved synteny of $a b c b 1 / A B C B 1$ and $a b c b 4 / A B C B 4$ regions in various species. Figure $\mathbf{S} \mathbf{2}$ with $\mathrm{Ct}$ values determined for housekeeping gene candidates in different embryo stages of zebrafish with $\mathrm{gPCR}$. The figure is supplementary to Table 1. Figure $\mathbf{S} \mathbf{3}$ with images of 120 hpf zebrafish embryos with abcb4 mRNA transcripts visualized using WISH. The figure is supplementary to Figure 2. Figure S4 with a standard curve used to determine the amount of RhB taken up by zebrafish embryos. The figure is supplementary to Figures 4 and 6 Figure S5 with images of Western blots with recombinant zebrafish Abcb4 protein obtained with the baculovirus expression system. Figure $\mathbf{S 6}$ with results of experiments proving the functionality of the used morpholinos.

\section{Abbreviations}

ABC: ATP-binding cassette; BCA: bicinchoninic acid assay; BLAST: basic alignment search tool; BSA: bovine serum albumin; CsA: cyclosporin A; DMSO: dimethyl sulfoxide; hpf: hours post fertilization; EPA: Environmental Protection Agency; ExPASy: Expert Protein Analysis System;

LC50: concentration that was lethal for $50 \%$ of embryos; Mb: megabases; MDR: multidrug resistance; MIQE: Minimum Information for Publication of Quantitative Real-Time PCR Experiments; MXR: multixenobiotic resistance; NCBI: National Center for Biotechnology Information;

PC: phosphatidylcholine; Pi: inorganic phosphate; GPCR: Quantitative realtime polymerase chain reaction; RACE: rapid amplification of CDNA ends; WISH: whole-mount in situ hybridization.

\section{Competing interests}

None of the contributing authors has any competing interests.

\section{Authors' contributions}

SF designed the study, performed experiments, analyzed data and wrote the paper. NK and KBM performed experiments, analyzed data and wrote the paper. MP, AMS and PW performed experiments and analyzed data. KS provided guidance to SF, analyzed data and wrote the paper. TL designed the study, provided guidance to SF, MP, KBM, AMS and PW, performed experiments, analyzed data and wrote the paper. All authors read, contributed feedback to, and approved the final manuscript.

\section{Authors' information}

Kristin Schirmer and Till Luckenbach are senior authors.

\section{Acknowledgements}

For technical support, we thank Mady Ahnert, Silke Aulhorn, Kathrin Schlüßler, Robert Suchsland (zebrafish toxicity assays) and Ute Lohse (DNA sequencing). Dr. Satoshi Endo provided help with calculating ATPase activity regression curves. We thank Dr. David Epel and Dr. Wibke Busch for comments on earlier versions of the manuscript. TL thanks Dr. Rita Triebskorn and Dr. Nicholas Foulkes for discussions and technical support in the early phase of the project. Partial funding was provided for this work by the Deutsche Forschungsgemeinschaft (DFG) (PI: TL) and the Ministry of Environment, Agriculture and Geology of Saxony (PI: KS). KBM is sponsored through the scholarship program of the German Federal Environmental Foundation (DBU).

\section{Author details}

${ }^{1}$ Department of Environmental Toxicology, Eawag, Swiss Federal Institute of Aquatic Science and Technology, 8600 Dübendorf, Switzerland. ${ }^{2}$ Department of Environmental Systems Sciences, ETH Zürich, Institute of Biogeochemistry and Pollutant Dynamics, 8092 Zürich, Switzerland. ${ }^{3}$ Department of Bioanalytical Ecotoxicology, UFZ - Helmholtz Centre for Environmental Research, 04318 Leipzig, Germany. ${ }^{4}$ Institute of Hydrobiology, Dresden University of Technology, 01062 Dresden, Germany. ${ }^{5}$ Laboratory of Environmental Toxicology, EPF Lausanne, School of Architecture, Civil and Environmental Engineering, 1015 Lausanne, Switzerland.

Received: 23 April 2013 Accepted: 7 June 2013

Published: 17 June 2013

\section{References}

1. Gottesman MM, Ambudkar SV: Overview: $A B C$ transporters and human disease. J Bioenerg Biomembr 2001, 33:453-458.

2. Kurelec B: The multixenobiotic resistance mechanism in aquatic organisms. Crit Rev Toxicol 1992, 22:23-43.

3. Schinkel AH: P-Glycoprotein, a gatekeeper in the blood-brain barrier. Adv Drug Deliv Rev 1999, 36:179-194.

4. Smital T, Sauerborn R, Pivcevic B, Krca S, Kurelec B: Interspecies differences in P-glycoprotein mediated activity of multixenobiotic resistance mechanism in several marine and freshwater invertebrates. Comp Biochem Physiol C Toxicol Pharmacol 2000, 126:175-186.

5. Luckenbach T, Epel D: ABCB- and ABCC-type transporters confer multixenobiotic resistance and form an environment-tissue barrier in bivalve gills. Am J Physiol Regul Integr Comp Physiol 2008, 294:R1919-R1929.

6. Higgins CF: Multiple molecular mechanisms for multidrug resistance transporters. Nature 2007, 446:749-757.

7. van Helvoort A, Smith AJ, Sprong H, Fritzsche I, Schinkel AH, Borst P, van Meer G: MDR1 P-glycoprotein is a lipid translocase of broad specificity, while MDR3 P-glycoprotein specifically translocates phosphatidylcholine. Cell 1996, 87:507-517.

8. Smith AJ, van Helvoort A, van Meer G, Szabo K, Welker E, Szakacs G, Varadi A, Sarkadi B, Borst P: MDR3 P-glycoprotein, a phosphatidylcholine translocase, transports several cytotoxic drugs and directly interacts with drugs as judged by interference with nucleotide trapping. I Biol Chem 2000, 275:23530-23539.

9. Frank NY, Margaryan A, Huang Y, Schatton T, Waaga-Gasser AM, Gasser M, Sayegh MH, Sadee W, Frank MH: ABCB5-mediated doxorubicin transport and chemoresistance in human malignant melanoma. Cancer Res 2005, 65:4320-4333

10. Hamdoun A, Epel D: Embryo stability and vulnerability in an always changing world. Proc Natl Acad Sci U S A 2007, 104:1745-1750. 
11. Toomey BH, Epel D: Multixenobiotic resistance in Urechis caupo embryos protection from environmental toxins. Biol Bull 1993, 185:355-364.

12. Scholz S, Fischer $S$, Gündel U, Küster E, Luckenbach T, Voelker D: The zebrafish embryo model in environmental risk assessment-applications beyond acute toxicity testing. Environ Sci Pollut Res Int 2008, 15:394-404.

13. Padilla S, Corum D, Padnos B, Hunter DL, Beam A, Houck KA, Sipes N, Kleinstreuer N, Knudsen T, Dix DJ, Reif DM: Zebrafish developmental screening of the ToxCast Phase I chemical library. Reprod Toxicol 2012, 33:174-187.

14. EPA: Computational toxicology research. [http://www.epa.gov/ncct/toxcast/]

15. Kokel D, Bryan J, Laggner C, White R, Cheung CY, Mateus R, Healey D, Kim S, Werdich AA, Haggarty SJ, Macrae CA, Shoichet B, Peterson RT: Rapid behavior-based identification of neuroactive small molecules in the zebrafish. Nat Chem Biol 2010, 6:231-237.

16. Volz DC, Belanger S, Embry M, Padilla S, Sanderson H, Schirmer K, Scholz S, Villeneuve D: Adverse outcome pathways during early fish development: a conceptual framework for identification of chemical screening and prioritization strategies. Toxicol Sci 2011, 123:349-358.

17. Annilo T, Chen ZQ, Shulenin S, Costantino J, Thomas L, Lou H, Stefanov S, Dean M: Evolution of the vertebrate $A B C$ gene family: analysis of gene birth and death. Genomics 2006, 88:1-11.

18. e! Ensemb: [http://www.ensembl.org]

19. Kane DA, Kimmel CB: The zebrafish midblastula transition. Development 1993, 119:447-456.

20. Thisse B, Thisse C: Fast release clones: A high throughput expression analysis. ZFIN direct data submission. 2004 [http://ffin.org/cgi-bin/webdriver?Mlval= aa-xpatselectapg\&query_results=true\&gene_name=abcb5\&searchtype=equals]

21. Frank NY, Pendse SS, Lapchak PH, Margaryan A, Shlain D, Doeing C, Sayegh $\mathrm{MH}$, Frank MH: Regulation of progenitor cell fusion by ABCB5 Pglycoprotein, a novel human ATP-binding cassette transporter. J Biol Chem 2003, 278:47156-47165.

22. Neyfakh AA: Use of fluorescent dyes as molecular probes for the study of multidrug resistance. Exp Cell Res 1988, 174:168-176.

23. Twentyman PR: Cyclosporins as drug resistance modifiers. Biochem Pharmacol 1992, 43:109-117.

24. Mayer U, Wagenaar E, Dorobek B, Beijnen JH, Borst P, Schinkel AH: Full blockade of intestinal P-glycoprotein and extensive inhibition of bloodbrain barrier P-glycoprotein by oral treatment of mice with PSC833. J Clin Invest 1997, 100:2430-2436.

25. Gekeler V, Ise W, Sanders KH, Ulrich WR, Beck J: The leukotriene LTD4 receptor antagonist MK571 specifically modulates MRP associated multidrug resistance. Biochem Biophys Res Commun 1995, 208:345-352.

26. Fischer S, Loncar J, Zaja R, Schnell S, Schirmer K, Smital T, Luckenbach T: Constitutive mRNA expression and protein activity levels of nine $A B C$ efflux transporters in seven permanent cell lines derived from different tissues of rainbow trout (Oncorhynchus mykiss). Aquat Toxicol 2011, 101:438-446.

27. Long Y, Li Q, Cui Z: Molecular analysis and heavy metal detoxification of ABCC1/MRP1 in zebrafish. Mol Biol Rep 2011, 38:1703-1711.

28. Litman T, Nielsen D, Skovsgaard T, Zeuthen T, Stein WD: ATPase activity of P-glycoprotein related to emergence of drug resistance in Ehrlich ascites tumor cell lines. Biochim Biophys Acta 1997, 1361:147-158.

29. Litman T, Zeuthen T, Skovsgaard T, Stein WD: Structure-activity relationships of P-glycoprotein interacting drugs: kinetic characterization of their effects on ATPase activity. Biochim Biophys Acta 1997, 1361:159-168.

30. Luckenbach T, Epel D: Nitromusk and polycyclic musk compounds as long-term inhibitors of cellular xenobiotic defense systems mediated by multidrug transporters. Environ Health Perspect 2005, 113:17-24.

31. Aller SG, Yu J, Ward A, Weng Y, Chittaboina S, Zhuo R, Harrell PM, Trinh YT, Zhang Q, Urbatsch IL, Chang G: Structure of P-glycoprotein reveals a molecular basis for poly-specific drug binding. Science 2009, 323:1718-1722.

32. Ueda K, Cardarelli C, Gottesman MM, Pastan I: Expression of a full-length cDNA for the human "MDR1" gene confers resistance to colchicine, doxorubicin, and vinblastine. Proc Natl Acad Sci U S A 1987, 84:3004-3008.

33. Fojo A, Akiyama S, Gottesman MM, Pastan I: Reduced drug accumulation in multiply drug-resistant human KB carcinoma cell lines. Cancer Res 1985, 45:3002-3007.

34. Horio M, Gottesman MM, Pastan I: ATP-dependent transport of vinblastine in vesicles from human multidrug-resistant cells. Proc Natl Acad Sci U S A 1988, 85:3580-3584.

35. Yang F, Chen Z, Pan J, Li X, Feng J, Yang H: An integrated microfluidic array system for evaluating toxicity and teratogenicity of drugs on embryonic zebrafish developmental dynamics. Biomicrofluidics 2011, 5:24115.
36. Sarkadi B, Price EM, Boucher RC, Germann UA, Scarborough GA: Expression of the human multidrug resistance CDNA in insect cells generates a high activity drug-stimulated membrane ATPase. J Biol Chem 1992, 267:4854-4858.

37. von Richter O, Glavinas H, Krajcsi P, Liehner S, Siewert B, Zech K: A novel screening strategy to identify $A B C B 1$ substrates and inhibitors. Naunyn Schmiedebergs Arch Pharmacol 2009, 379:11-26.

38. Saeki T, Ueda K, Tanigawara Y, Hori R, Komano T: Human P-glycoprotein transports cyclosporin A and FK506. J Biol Chem 1993, 268:6077-6080.

39. Balk F, Blok H, Salvito D: Environmental risks of musk fragrance ingredients. In Pharmaceuticals and Personal Care Products in the Environment - Scientific and Regulatory Issues. Edited by Daughton CG, Jones-Lepp TL. Washington, DC: American Chemical Society; 2001:168-190.

40. Goto T, Holzinger F, Hagey LR, Cerre C, Ton-Nu HT, Schteingart CD, Steinbach JH, Shneider BL, Hofmann AF: Physicochemical and physiological properties of 5alpha-cyprinol sulfate, the toxic bile salt of cyprinid fish. J Lipid Res 2003, 44:1643-1651.

41. Park $D$, Haldi $M$, Seng $W L$ : Zebrafish: a new in vivo model for identifying P-glycoprotein efflux modulators. In Zebrafish: Methods for Assessing Drug Safety and Toxicity. Edited by McGrath P. Hoboken, NJ, USA: John Wiley \& Sons, Inc; 2011:177-190.

42. Epel D, Luckenbach T, Stevenson CN, Macmanus-Spencer LA, Hamdoun A, Smital T: Efflux transporters: newly appreciated roles in protection against pollutants. Environ Sci Technol 2008, 42:3914-3920.

43. Carlsson G, Norrgren L: Synthetic musk toxicity to early life stages of zebrafish (Danio rerio). Arch Environ Contam Toxicol 2004, 46:102-105.

44. Westerfield M: The Zebrafish Book. A Guide for the Laboratory Use of Zebrafish (Danio rerio). 4th edition. Eugene, OR: University of Oregon Press; 2000.

45. Nagel R: DarT: The embryo test with the zebrafish Danio rerio - a general model in ecotoxicology and toxicology. ALTEX 2002, 19:38-48.

46. ExPASy: Bioinformatics Resource Portal. [http://expasy.org]

47. Q Gene: Software for QTL Data Exploration. [http://www.qgene.org/]

48. Muller PY, Janovjak H, Miserez AR, Dobbie Z: Processing of gene expression data generated by quantitative real-time RT-PCR. Biotechniques 2002, 32:1372-1374. 1376, 1378-1379.

49. Simon P: Q-Gene: processing quantitative real-time RT-PCR data. Bioinformatics 2003, 19:1439-1440.

50. Bustin SA, Benes V, Garson JA, Hellemans J, Huggett J, Kubista M, Mueller R, Nolan T, Pfaffl MW, Shipley GL, Vandesompele J, Wittwer CT: The MIQE guidelines: minimum information for publication of quantitative realtime PCR experiments. Clin Chem 2009, 55:611-622.

51. Thisse C, Thisse B: High-resolution in situ hybridization to whole-mount zebrafish embryos. Nat Protoc 2008, 3:59-69.

52. Yeheskely-Hayon D, Regev R, Katzir H, Eytan GD: Competition between innate multidrug resistance and intracellular binding of rhodamine dyes. FEBS J 2009, 276:637-648.

53. Essodaigui M, Broxterman HJ, Garnier-Suillerot A: Kinetic analysis of calcein and calcein - acetoxymethylester efflux mediated by the multidrug resistance protein and P-glycoprotein. Biochemistry 1998, 37:2243-2250.

54. Hollo Z, Homolya L, Davis CW, Sarkadi B: Calcein accumulation as a fluorometric functional assay of the multidrug transporter. Biochim Biophys Acta 1994, 1191:384-388.

55. Robey RW, Zhan ZR, Piekarz RL, Kayastha GL, Fojo T, Bates SE: Increased MDR1 expression in normal and malignant peripheral blood mononuclear cells obtained from patients receiving depsipeptide (FR901228, FK228, NSC630176). Clin Cancer Res 2006, 12:1547-1555.

56. Schreiber R, Altenburger R, Paschke A, Schuurmann G, Kuster E: A novel in vitro system for the determination of bioconcentration factors and the internal dose in zebrafish (Danio rerio) eggs. Chemosphere 2009, 77:928-933.

57. Germann UA: Baculovirus-mediated expression of human multidrug resistance cDNA in insect cells and functional analysis of recombinant Pglycoprotein. Methods Enzymol 1998, 292:427-441.

58. Kurelec B, Smital T, Pivcevic B, Eufemia N, Epel D: Multixenobiotic resistance, P-glycoprotein, and chemosensitizers. Ecotoxicology 2000, 9:307-327.

59. ZFIN Zebrafish Nomenclature Guidelines: [http://wiki.zfin.org/display/ general/ZFIN+Zebrafish+Nomenclature+Guidelines]

doi:10.1186/1741-7007-11-69

Cite this article as: Fischer et al:: Abcb4 acts as multixenobiotic transporter and active barrier against chemical uptake in zebrafish (Danio rerio) embryos. BMC Biology 2013 11:69. 